EGU21-1131, updated on 20 Aug 2021

https://doi.org/10.5194/egusphere-egu21-1131

EGU General Assembly 2021

(c) Author(s) 2021. This work is distributed under

the Creative Commons Attribution 4.0 License.

\title{
Applying BEL1D for transient electromagnetic sounding inversion
}

\author{
Arsalan Ahmed ${ }^{1}$, Hadrien Michel ${ }^{1,2,3}$, Wouter Deleersnyder $^{1,4}$, David Dudal $^{4}$, and Thomas \\ Hermans $^{1}$ \\ ${ }^{1}$ Ghent University, Department of Geology, Ghent, Belgium \\ ${ }^{2}$ University of Liege, Urban and Environmental Engineering Department, Faculty of Applied Sciences, Liege, Belgium \\ ${ }^{3}$ F.R.S.-FNRS(Fonds de la Recherche Scientifique), Brussels, Belgium \\ ${ }^{4}$ KU Leuven Campus Kortrijk - Kulak, Department of Physics, Kortrijk, Belgium
}

Accurate subsurface imaging through geophysics is of prime importance for many geological and hydrogeological applications. Recently, airborne electromagnetic methods have become more popular because of their potential to quickly acquire large data sets at relevant depths for hydrogeological applications. However, the solution of inversion of airborne EM data is not unique, so that many electrical conductivity models can explain the data. Two families of methods can be applied for inversion: deterministic and stochastic methods. Deterministic (or regularized) approaches are limited in terms of uncertainty quantification as they propose one unique solution according to the chosen regularization term. In contrast, stochastic methods are able to generate many models fitting the data. The most common approach is to use Markov chain Monte Carlo (McMC) Methods. However, the application of stochastic methods, even though more informative than deterministic ones, is rare due to a quite high computational cost.

In this research, the newly developed approach named Bayesian Evidential Learning 1D imaging (BEL1D) is used to efficiently and stochastically solve the inverse problem. BEL1D is combined to SimPEG: an open source python package, for solving the electromagnetic forward problem. BEL1D bypasses the inversion step, by generating random samples from the prior distribution with defined ranges for the thickness and electrical conductivity of the different layers, simulating the corresponding data and learning a direct statistical relationship between data and model parameters. From this relationship, BEL1D can generate posterior models fitting the field observed data, without additional forward model computations. The output of BEL1D shows the range of uncertainty for subsurface models. It enables to identify which model parameters are the most sensitive and can be accurately estimated from the electromagnetic data.

The application of BEL1D together with SimPEG for stochastic transient electromagnetic inversion is a very efficient approach, as it allows to estimate the uncertainty at a limited cost. Indeed, only a limited number of training models (typically a few thousands) is required for an accurate prediction. Moreover, the computed training models can be reused for other predictions, considerably reducing the computation cost when dealing with similar data sets. It is thus a promising approach for the inversion of dense data set (such as those collected in airborne surveys). In the future, we plan on relaxing constraints on the model parameters to go towards 
interpretation of EM data in coastal environment, where transition can be smooth due to salinity variations.

Keywords : EM, Uncertainty, 1D imaging, BEL1D, SimPEG 